\title{
LA PHYSIQUE NON-HERMITIENNE DES CAVITÉS OPTIQUES
}

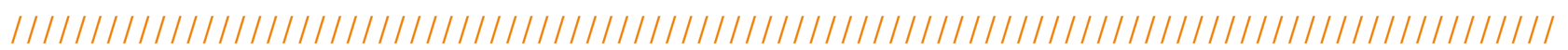

\section{Philippe LALANNE}

Laboratoire Photonique, Numérique et Nanosciences (LP2N), IOGS, Université de Bordeaux, 33400 Talence philippe.lalanne@institutoptique.fr

Les nano-cavités, plus généralement les cavités électromagnétiques, sont des systèmes ouverts qui ne conservent pas l'énergie : leurs « modes propres ", c'est-à-dire leurs résonances naturelles, soulèvent des difficultés théoriques qui ont longtemps freiné le développement d'une théorie modale. Des progrès récents importants viennent d'être enregistrés. Ils impactent la façon de modéliser et comprendre les nano-résonateurs. Ils prédisent aussi des effets contre-intuitifs qui conduisent à revisiter des grands classiques du confinement, comme l'effet Purcell ou la théorie de perturbation des cavités, souvent indûment considérés dans un cadre hermitien.
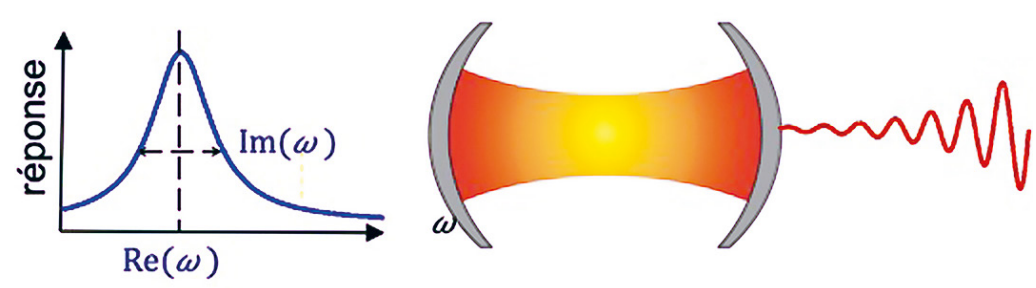

https://doi.org/10.1051/photon/202010046

Article publié en accès libre sous les conditions définies par la licence Creative Commons Attribution License CC-BY (http://creativecommons.org/licenses/by/4.0), qui autorise sans restrictions l'utilisation, la diffusion, et la reproduction sur quelque support que ce soit, sous réserve de citation correcte de la publication originale.

\section{LES CAVITÉS OPTIQUES}

Qu'il s'agisse d'un diapason, d'un trou noir ou d'une microcavité, les résonances sont observées dès lors qu'une onde interagit avec des structures; elles sont souvent utilisées à dessein ou parfois combattues pour leurs effets néfastes. Quelle qu'en soit leur origine, elles génèrent des champs bien plus intenses que le champ excitateur, en les localisant spatialement et les stockant temporellement. L'exaltation de champ est au cœur de presque toutes les applications des résonateurs.
Deux paramètres caractéristiques occupent une place importante dans la physique et les applications des cavités, le facteur de qualité $Q$ et le volume du mode $V$. Nous connaissons tous les modes stationnaires des cavités Fabry-Perot ou les modes de galerie des microsphères qui permettent d'atteindre des facteurs de qualité très élevés (parfois $>10^{8}$ ) dans des volumes centimétriques $\left(V \sim 10^{12} \lambda^{3}\right)$. $Q$ et $V$ seront définis précisément plus loin, mais pour l'instant, retenons simplement que $Q$ caractérise la dynamique temporelle et $V$ la localisation spatiale.

Note au lecteur : Des erreurs se sont glissées dans la version initiale de cet article. Elles sont décrites dans la notice de correction. Cette nouvelle version publiée le 3 avril 2020 contient toutes les corrections.

Les cavités ont connu une évolution rapide au cours des deux dernières décennies. À la fin du siècle dernier, les chercheurs ont rivalisé d'imagination pour réaliser des microcavités diélectriques qui ont permis de confiner la lumière pendant presque 1 nanoseconde $\left(Q=10^{6}\right)$ sur des volumes de l'ordre de quelques $\lambda^{3}$ seulement. Un second changement de paradigme s'est produit peu après avec l'avènement des nanoantennes plasmoniques, léquivalent optique des antennes électromagnétiques basses fréquences, dont les champs évanescents en surface des métaux offrent des confinements à des échelles bien inférieures à la longueur d'onde. $Q$ est sacrifié dans ces nanoantennes, mais les volumes 
deviennent si petits que des exaltations importantes restent possibles avec peu de photons. Les travaux actuels portent sur les nano-cavités (nanogap antenna dans la figure 1) qui confinent la lumière dans des gaps nanométriques. La dernière-née des géométries, qui n'est pas répertoriée figure 1, a permis d'atteindre un degré de confinement exceptionnel en localisant la lumière autour d'une minuscule protubérance constituée semble-t-il d'un seul atome métallique stabilisé sous irradiation laser dans un gap nanométrique. Comme $V$ a été estimé à moins d'un nanomètre cube à partir de mesures de vibrations de molécules uniques, ces cavités ont été appelées « picocavités » [1].

\section{MODES QUASI-NORMAUX DES SYSTÈMES \\ NON-HERMITIENS}

Les résonateurs sont des systèmes ouverts, l'énergie $y$ entre et s'en échappe perpétuellement. L'énergie n'est donc pas une quantité conservée et les «états propres » ne sont pas des ondes stationnaires. Leur intensité décroit exponentiellement dans le temps, par absorption (parfois) ou par rayonnement (toujours) [2]. On parle de physique non-hermitienne. Mathématiquement, cela se traduit par le fait que les modes (ou états) possèdent des fréquences propres $\tilde{\omega}$ (ou énergies $\hbar \tilde{\omega}$ ) complexes (notées avec un tilde)

$$
\tilde{\omega}=\omega-i \Gamma / 2
$$

$\Gamma$ étant le taux de décroissance, c'est-à-dire l'inverse de la durée de vie du mode $\tau=1 / \Gamma$. Le signe moins de l'équation provient de la convention en $\exp (-i \tilde{\omega} t)$ et le facteur 2 tient compte de la différence entre décroissances en amplitude ou énergie.

En physique théorique, ces «états propres » sont souvent appelés modes quasi-normaux (QNMs en abrégé) ou résonances, pour souligner leur différence marquée avec les modes normaux des systèmes hermitiens. Les QNMs sont les solutions harmoniques d'opérateurs différentiels linéarisés, ici les équations de Maxwell sans source

$$
\nabla \times \tilde{\mathbf{E}}=i \widetilde{\omega} \mu_{0} \tilde{\mathbf{H}}, \nabla \times \tilde{\mathbf{H}}=-i \tilde{\omega} \varepsilon(\widetilde{\omega}) \tilde{\mathrm{E}} .(2)
$$

Pour bien comprendre l'impact des fréquences complexes, regardons d'abord le cas des cavités hermitiennes idéales, fermées et sans dissipation. $\widetilde{\omega}$ et $\varepsilon$ sont alors tous deux des réels, et les équations de Maxwell impliquent que $\tilde{\mathbf{E}}$ (ou $\tilde{\mathbf{H}}$ ) soit purement réel (ou imaginaire). Le champ électrique $\operatorname{Re}(\tilde{\mathbf{E}} \exp (-i \tilde{\omega} t))$ $=\tilde{\mathbf{E}} \cos (\tilde{\omega} t) \mathrm{du}$ mode est alors stationnaire ; il peut être représenté par une fonction séparable de $t$ et de $\boldsymbol{r}$. Des exemples classiques étant le résonateur Fabry-Perot avec deux miroirs parfaits, ou une corde sans dissipation maintenue à ses deux extrémités. En revanche, pour les systèmes dissipatifs, $\varepsilon, \widetilde{\omega}, \tilde{\mathbf{E}}$ et $\tilde{\mathbf{H}}$ deviennent tous complexes, et chaque composante $i$ du champ réel, comme la composante $x$, $\left|\tilde{\mathbf{E}}_{\mathrm{x}}\right| \cos \left(\operatorname{Re}(\tilde{\omega}) \mathrm{t}+\phi_{x}\right) \exp (-t / \tau)$ n'est plus factorisable en un produit de deux fonctions qui dépendent $d u$ temps et de l'espace, car le facteur de phase $\phi_{x}$ dépend de l'espace, préfigurant la présence d'ondes progressives dans le champ. La présence de cette phase n'est pas anodine : c'est la signature de la non-hermiticité. On verra par la suite qu'elle a de lourdes conséquences sur la physique des nanorésonateurs.

\section{NORMALISATION \\ ET RECONSTRUCTION DANS}

\section{LA BASE DES ÉTATS PROPRES}

Le caractère non-hermitien des QNMs a une conséquence épineuse: la divergence exponentielle de leur champ à l'extérieur de la structure. Cela est facile à réaliser en considérant un résonateur placé dans le vide et le comportement asymptotique de son champ lointain en $r^{-1} \exp i(t-r / c)$. Le signe de $\operatorname{Im}(\widetilde{\omega})$ étant fixé par la décroissance exponentiellement dans le temps, le signe moins dans $(t-r / c)$ induit une divergence exponentielle aux grandes distances. Cette divergence

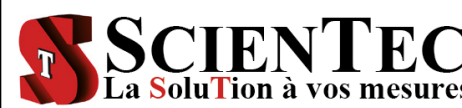

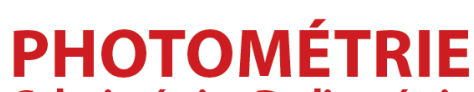

Colorimétrie - Radiométrie MESURE ETÉVALUATION DETOUTES LES SOURCES LUMINEUSES

Éclairement, luminance, chromaticité, indice de rendu des couleurs IRC, température de couleur, longueurs d'ondes crête et dominante, valeurs spectrales, pureté d'excitation...
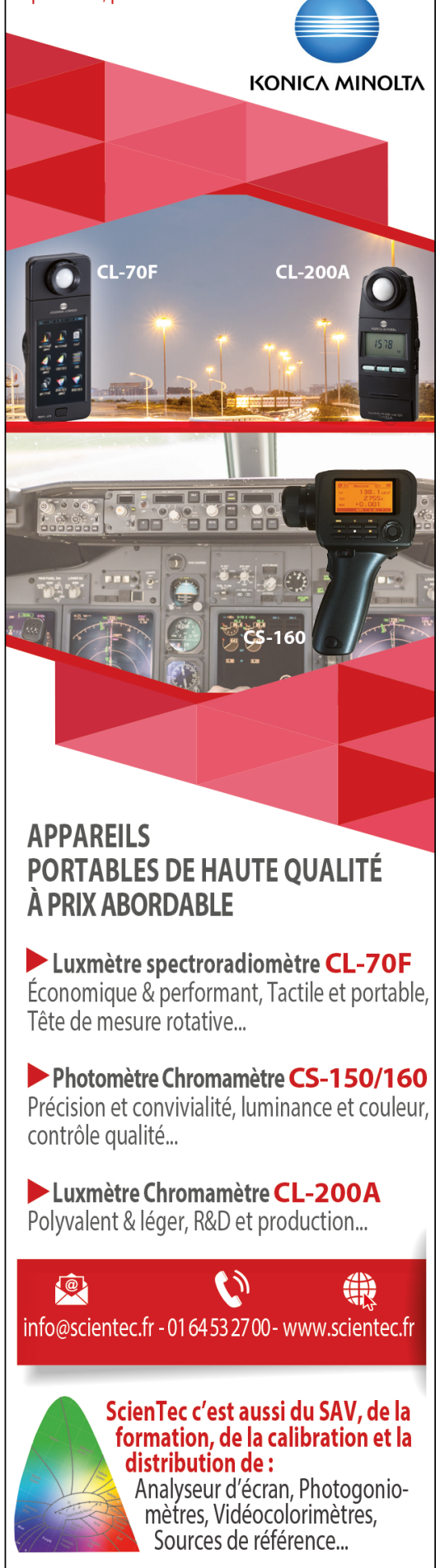


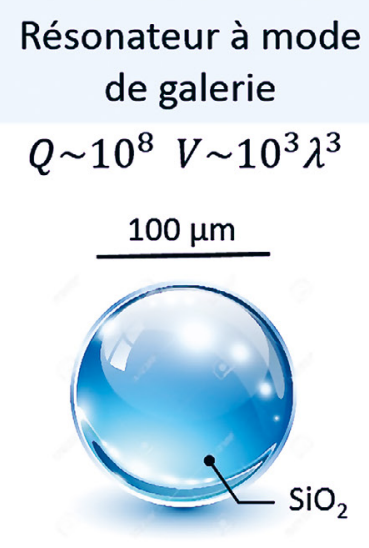

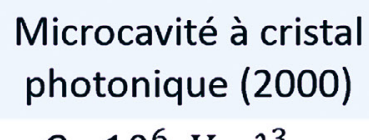

$$
Q \sim 10^{6} V \sim \lambda^{3}
$$

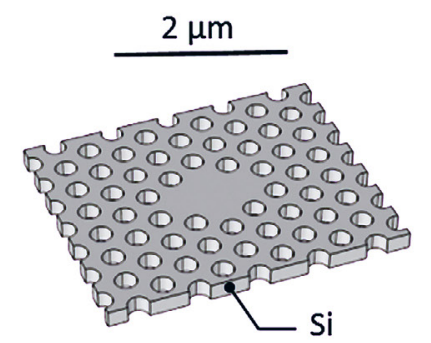

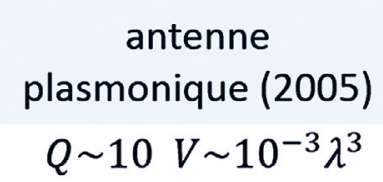

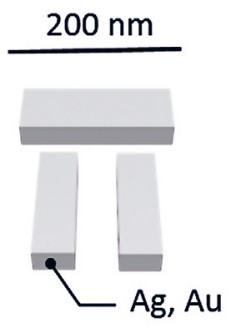

cavité à gap nanométrique (2012) $Q \sim 10 V \sim 10^{-6} \lambda^{3}$

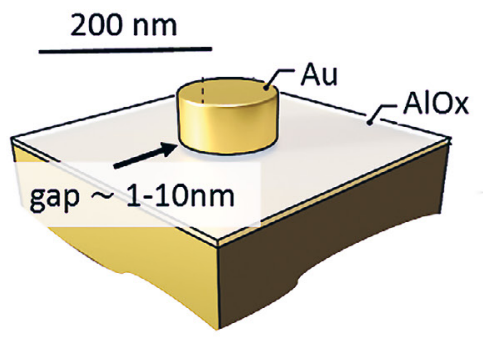

a longtemps été un frein au développement d'une théorie modale des résonateurs, semblable à celle des guides d'ondes en optique guidée. C'est d'autant plus préjudiciable que les modes résonnants sont au cœur de la physique et des applications des résonateurs.

Pourtant la plupart des logiciels, y compris les logiciels commerciaux, calculent depuis longtemps les QNMs, en résolvant les équations (2) comme un problème aux vecteurs et valeurs propres [2]. Le problème ne provient donc pas du calcul des modes, mais de leur normalisation nécessaire à la prédiction de leur taux d'excitation par une source et donc d'une représentation de l'évolution du système sur la base des « états propres ». Les choses évoluent très vite aujourd'hui.

Les premiers travaux sur la reconstruction de champ dans la base des QNMs ont commencé dans les années 30 en mécanique ondulatoire pour les cas de barrières de potentiel de hauteur finie 1D ou 3D sphériques, puis ils ont été repris en électromagnétisme dans les années 70 avec les mêmes distributions de « potentiel ». Depuis longtemps, on sait donc traiter des cas simples pour lesquels le champ et sa divergence sont connus analytiquement. Au début des années 2010, deux travaux importants par deux équipes françaises ont ouvert les portes vers l'analyse de géométries
Figure 1.

Structures emblématiques qui ont marqué l'histoire du confinement de la lumière.

De gauche à droite, sphères ou disques optiques diélectriques à mode de galerie ; microcavités à cristaux photoniques dans des films semiconducteurs; antennes plasmoniques à nanoparticules métalliques; nano-cavités métallo-diélectriques avec des gaps nanométriques.

modernes, complexes aussi, par exemple comme celles de la figure 1. C'est ainsi qu'une approche théorique générale a été élaborée, permettant de normaliser les modes de résonateurs arbitraires, 3D, constitués de milieux dispersifs ou anisotropes, pas nécessairement placés dans le vide mais déposés sur un substrat par exemple; le second résultat a établi que pour les résonateurs qui ne sont pas dans un milieu homogène, la base des QNMs n'était pas complète, mais pouvaient être enrichie d'autres modes garantissant la complétude.

Depuis, le nombre de travaux sur les QNMs en électromagnétisme est en forte progression. Pas moins d'une douzaine de groupes de théoriciens travaillent aujourd'hui sur le sujet dans le monde. L'école Française d'optique électromagnétique, avec ses travaux pionniers sur le sujet dans le cadre de la diffraction électromagnétique par les réseaux [3], est aujourd'hui fédérée en grande partie par le projet ANR «Résonance », et compte cinq équipes CNRS qui sont à la pointe de la recherche mondiale du domaine.

La figure 2 montre un exemple de reconstruction qui n'est possible que depuis peu. Il s'agit d'une antenne plasmonique dont la résonance est très sensible à la présence de nano-objets étrangers. L'antenne est éclairée par un pulse visible de durée 10 fs. La reconstruction modale est obtenue à partir de seulement 3 modes, dont le calcul ne nécessite que quelques minutes avec un ordinateur de bureau, et de leurs coefficients d'excitation $a_{i}(t)$ dont l'expression analytique ne nécessite qu'un calcul intégral de recouvrement entre le mode normalisé et le pulse excitateur. Elle est comparée à une reconstruction « exacte », obtenue au moyen de la méthode de référence pour ce genre de calcul aujourd'hui, la FDTD qu'il n'est plus besoin de présenter. Il est important de réaliser que le calcul FDTD nécessite plusieurs heures et un ordinateur doté d'une mémoire importante, et que surtout, il doit être entièrement recommencé, dès lors que le moindre paramètre du pulse incident, comme sa polarisation, sa direction ou sa durée, est changé. En revanche, l'approche modale ne demande aucun calcul supplémentaire, les modes étant intrinsèques et déjà calculés. Elle est donc beaucoup plus efficace, mais 
elle est surtout beaucoup plus parlante avec ses expressions analytiques et limpides, des coefficients d'excitation qui rendent transparente l'origine du battement. C'est là toute la force des méthodes modales par rapport à des méthodes complètement numériques.

Il reste de nombreux problèmes théoriques à comprendre, des raffinements à apporter. Mathématiquement, des difficultés naissent pour des géométries pour lesquelles le plan des fréquences complexes n'est pas seulement rempli de pôles (les QNMs), mais contient aussi des branches. aux premiers travaux au début du siècle dernier sur la théorie de la perturbation des cavités initialement développée pour interpréter des mesures radiofréquences de l'absorption résiduelle de certains matériaux diélectriques. Pour réaliser combien nous sommes imprégnés par cette physique, creusons un peu autour de la signification de $Q$ et $V$, les deux paramètres caractéristiques du degré de confinement spatial et temporel au cœur de la physique et des applications des cavités, comme l'effet Purcell que nous examinerons dans un second temps.

\section{Les premiers travaux sur la reconstruction} de champ dans la base des QNMs ont commencé dans les années 30 en mécanique ondulatoire pour les cas de barrières de potentiel de hauteur finie 1D ou 3D sphériques, puis ils ont été repris en électromagnétisme dans les années 70 avec les mêmes distributions de « potentiel ».
Malgré cela, les toutes premières reconstructions convaincantes viennent d'être obtenues pour des géométries complexes, telles que les réseaux de diffraction avec leurs séries de fréquences de coupure qui conduisent à autant de branches, les nanocavités sur substrat, en particulier quand le substrat peut guider la lumière ou supporter des ondes de surface et que la lumière fuit dans ces modes guidés... Les tous premiers logiciels voient aussi le jour.

\section{PHYSIQUE NON-HERMITIENNE : Q ET V}

Notre conceptualisation actuelle du confinement est issue d'une longue tradition de pensée dans le cadre restrictif de la physique hermitienne, tradition qui remonte
En génie électrique, le facteur de qualité est communément défini [4] comme le rapport entre l'énergie emmagasinée dans la cavité et la puissance dissipée par cycle. Cette définition est légitime pour des modèles simplifiés remplaçant des systèmes distribués par des éléments discrets (circuits RLC), mais de manière générale, comment définir l'énergie emmagasinée, $\int\left(\varepsilon|\tilde{\mathbf{E}}|^{2}+\mu_{0}|\tilde{\mathbf{H}}|^{2}\right) d^{3} \boldsymbol{r}$, dans le mode d'un système ouvert avec un champ qui diverge exponentiellement ? Par exemple, pour la cavité à cristaux photoniques décrit à la figure 1, quel volume d'intégration doit-on considérer pour éviter la divergence obtenue pour une intégration sur tout l'espace? La théorie QNM des systèmes non-Hermitiens donne une
Lasers Accordables

Wattmètres

Contrôleurs de Polarisation

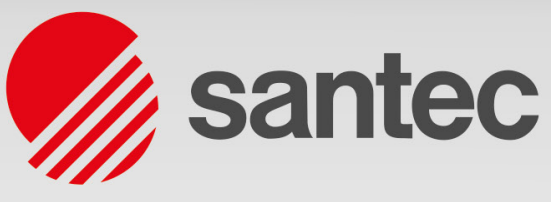

La Pensée Précise

\author{
SANTEC CORPORATION \\ www.santec.com
}

santec-emea@santec.com 
définition univoque,

$$
Q=-\frac{1}{2} \operatorname{Re}(\widetilde{\omega}) / \operatorname{Im}(\widetilde{\omega}),
$$

applicable indifféremment aux systèmes qui dissipent leur énergie imperceptiblement ou significativement à l'échelle d'un cycle. Elle permet de montrer que cette définition coïncide bien avec la définition énergétique communément admise, mais uniquement dans la limite des systèmes à faibles pertes. Dans le cas des fortes pertes, elle montre que définition énergétique et notion d'énergie emmagasinée perdent tout pouvoir prédictif ou intuitif [2].

Le volume modal, initialement introduit dans le cadre de la théorie de la perturbation des cavités radiofréquences, a été surtout popularisé par les études d'électrodynamique quantique en cavité [5]. Sa signification peut être comprise en considérant une cavité perturbée par un minuscule corps étranger, une molécule par exemple qui peut être représentée par un dipôle ponctuel polarisé. D'une part, la molécule perturbe les modes de cavité, modifiant ainsi leurs fréquences complexes, et d'autre part, les modes polarisent la molécule. La force de cette interaction mutuelle, qui dépend de la position $\boldsymbol{r}$ et de la direction de polarisation $\boldsymbol{u}$ du dipôle, mesure la capacité du mode à renforcer l'interaction entre la lumière et la matière ; c'est le volume modal.

La communauté a commencé avec des travaux sur des cavités présentant de très grands facteurs de qualité et a naturellement adopté une définition de $\mathrm{V}$, dérivée dans le cadre hermitien des faibles pertes et basée sur la densité d'énergie électromagnétique, $V_{E}(\boldsymbol{r})=\frac{\int\left[\varepsilon|\tilde{\mathbf{E}}|^{2}+\mu_{0}|\tilde{\mathbf{H}}|^{2}\right] d^{3} \boldsymbol{r}}{2 \varepsilon(\boldsymbol{r})|\tilde{\mathbf{E}}(\boldsymbol{r}) \cdot \mathbf{u}|^{2}}$. C'était aussi la seule expression disponible à l'époque. Dans cette expression qui suggère intuitivement que l'interaction la plus forte est mise en œuvre au maximum d'intensité de champ du mode aligné sur $\mathbf{u}$ (quand $|\tilde{\boldsymbol{E}}(\boldsymbol{r}) \cdot \mathbf{u}|^{2}$ est maximum), la phase du mode, $\phi_{i}(r)$ est complètement absente. Ainsi, toute manifestation de la physique non-hermitienne (voir plus haut) est négligée. Initialement, la communauté a commencé par étudier des cavités à forts $Q$ et a pu s'accommoder de cette définition, même si elle conduisait parfois à des prédictions erronées, comme celle concernant le changement de facteur de qualité induit par une perturbation. Avec l'avènement des nanoantennes plasmoniques, l'approche hermitienne est devenue indéfendable; les travaux se sont intensifiés pour aboutir à la définition

Figure 2.

Réponse d'une nanoantenne plasmonique composée de trois nano-bâtonnets d'or à une impulsion dans le visible. Les battements (courbe bleue) calculés avec la méthode de référence, la FDTD (finite-difference-time-domain), sont reproduits fidèlement et expliqués comme la somme des contributions individuelles de 3 modes avec des coefficients d'excitation donnés par $a_{i}(t), i=1,2,3$. Sur cet exemple, l'approche modale est bien plus rapide (100-1000x) que la FDTD, mais surtout elle explique de façon transparente l'origine physique du battement. La simulation est faite avec le logiciel MAN (Modal Analysis of Nanoresonators), LP2N. d'un volume de mode dans un cadre général non-Hermitien [2],

$$
\tilde{V}(\boldsymbol{r})=\frac{\int\left[\varepsilon \tilde{\boldsymbol{E}}^{2}-\mu_{0} \tilde{\boldsymbol{H}}^{2}\right] d^{3} \boldsymbol{r}}{2 \varepsilon(\boldsymbol{r})(\tilde{\boldsymbol{E}}(\boldsymbol{r}) \cdot \boldsymbol{u})^{2}},
$$

une grandeur à valeur complexe dans laquelle la phase du mode apparaît explicitement, notamment au dénominateur avec un terme en $\exp (2 i \phi(\boldsymbol{r}))$. Le facteur deux n'est pas une coïncidence, car chaque mécanisme individuel de l'interaction mutuelle est responsable d'un retard $\phi(\boldsymbol{r})$. L'interprétation de l'équation (4), et notamment de la normalisation qui apparait au numérateur, n'est pas simple. Il est cependant facile et réconfortant de vérifier que, dans la limite des faibles pertes, $\tilde{V}(\boldsymbol{r}) \rightarrow V_{E}(\boldsymbol{r})$ [2].

Initialement considéré comme une abstraction mathématique, le concept d'un volume complexe avec une partie imaginaire liée à la nature non-hermitienne des systèmes ouverts a progressivement gagné ses lettres de noblesse en montrant sa pertinence pour modéliser les mécanismes fondamentaux $\mathrm{du}$ confinement. Le concept a récemment reçu un fort support expérimental avec les premières mesures qui ont permis d'accéder à $\operatorname{Re}(\tilde{V})$ et $\operatorname{Im}(\tilde{V})$ individuellement. L'expérience effectuée en scannant une sonde

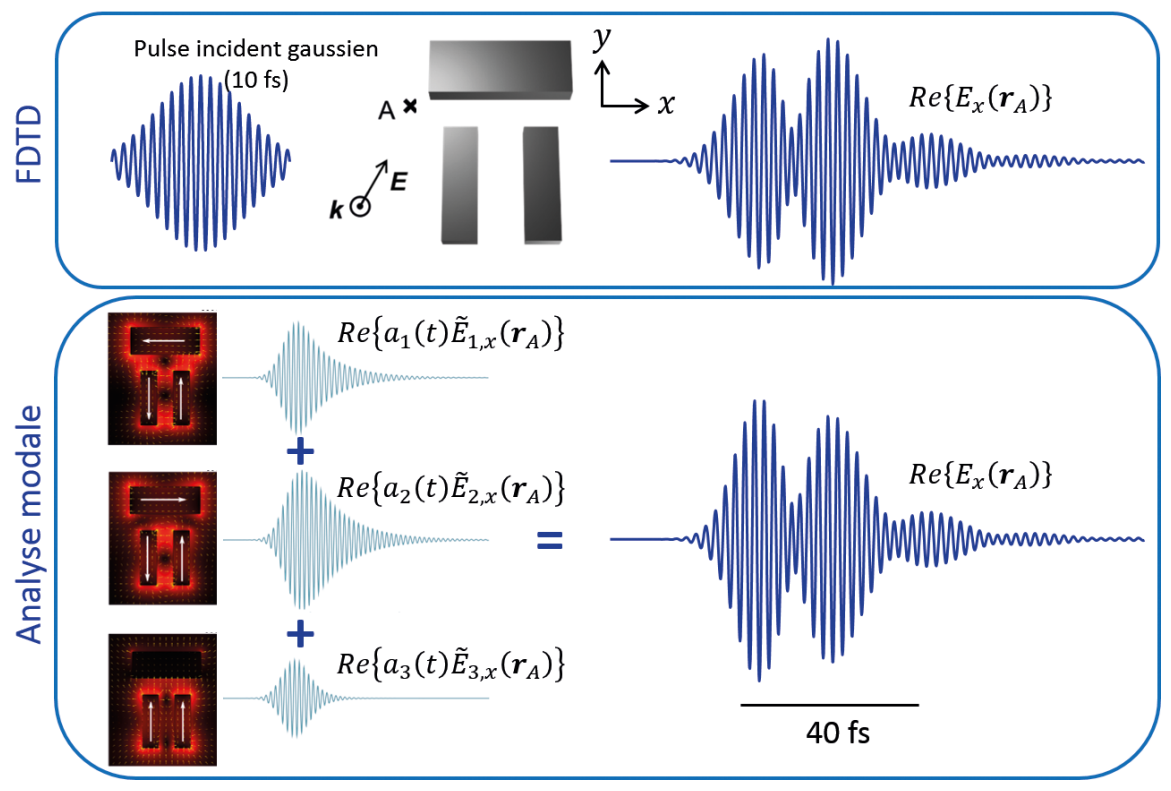


locale diélectrique dans le champ proche d'une cavité à cristal photonique propose de fait une méthode directe et systématique pour cartographier $\tilde{V}(\boldsymbol{r})$, lui donnant ainsi un sens concret. de l'espace. Temps et espace s'imbriquent dans la densité locale d'états, de la même manière qu'ils s'imbriquent pour les champs des modes non-hermitiens (voir plus haut).

Q et Ṽapparaissent dans de nombreux effets, le plus célèbre étant sans doute l'effet Purcell [5], qui est une manifestation de la modification de la densité locale d'états électromagnétiques (LDOS) par les modes de cavités

$Q$ et $\tilde{V}$ apparaissent dans de nombreux effets, le plus célèbre étant sans doute l'effet Purcell [5], qui est une manifestation de la modification de la densité locale d'états électromagnétiques (LDOS) par les modes de cavités. En pratique l'effet se traduit par une modification du taux d'émission spontanée d'émetteurs quantiques en cavité. Dans la vision hermitienne traditionnelle, les dépendances spatiale et temporelle sont factorisées dans l'expression classique du facteur de Purcell qui est proportionnel à $Q / V$. Ce partitionnement de la LDOS, largement adopté en particulier par la communauté travaillant sur l'électrodynamique quantique, est clair quand la durée de vie du mode $\tau=-2 / \operatorname{Im}(\tilde{\omega})$ est beaucoup plus grande que la période d'oscillation du champ $\operatorname{Re}(\tilde{\omega})$. Mais dans les cas de fortes pertes (énergie dissipée en quelques oscillations ou même une oscillation), comment partitionner la LDOS entre l'espace et le temps devient bien moins trivial. La théorie QNM [2] montre que le facteur de Purcell ne peut plus être factorisé comme le produit de deux fonctions dépendant respectivement du temps et
Cela conduit à des effets contre-intuitifs, non anticipés dans le cadre hermitien et favorisés par des valeurs conséquentes de $\operatorname{Im}(\tilde{V})$ par rapport à $\operatorname{Re}(\tilde{V})$. Par exemple, des valeurs du rapport $\operatorname{Im}(\tilde{V}) / \operatorname{Re}(\tilde{V})$ proches de l'unité conduisent à un profil spectral de type Fano pour la modification du taux d'émission, par opposition au profil lorentzien classique des systèmes hermitiens. Ces modes particulièrement non-hermitiens peuvent en outre contribuer négativement à la densité locale des états optiques, en exerçant une force réactive de friction sur l'émetteur qui en augmente la durée de vie, au lieu de la diminuer. Ces deux effets, qui viennent d'être mis en évidence expérimentalement, sont illustrés à la figure 3.

Le rapport $\operatorname{Im}(\tilde{V}) / \operatorname{Re}(\tilde{V})$ est enraciné dans de nombreux effets fondamentaux de l'interaction lumière-matière. Par exemple, il est prédit que, pour certains modes, l'image habituelle $\mathrm{du}$ couplage fort entre excitons et plasmons est fortement impacté par $\operatorname{Im}(\tilde{V})$, au point de complètement brouiller la frontière traditionnelle entre les régimes de couplages faible et fort, notamment en réfutant l'existence d'un point exceptionnel où les énergies des deux modes polaritoniques couplés fusionnent
YOUR EXPERT IN

PHOTONIC

INTEGRATION
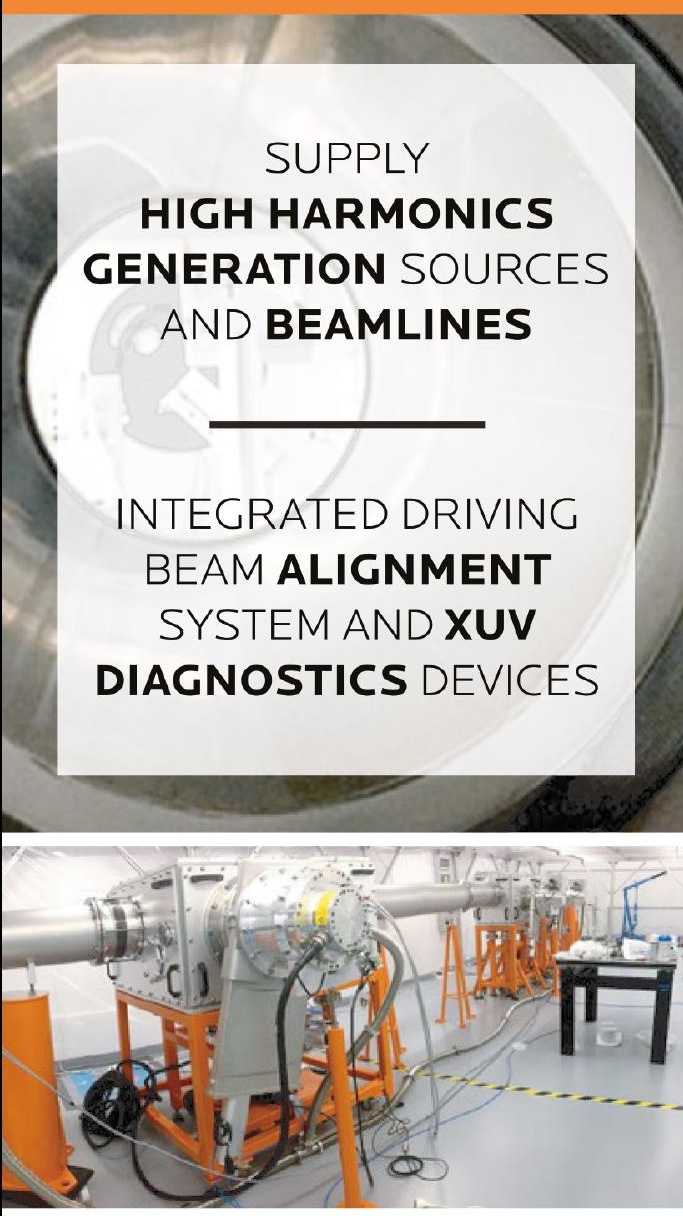

LASER

PHOTOMETRY

RADIOMETRY

COLORIMETRY

SPECTROSCOPY
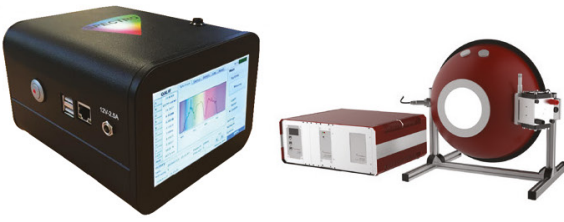

INTEGRATOR PARTNER

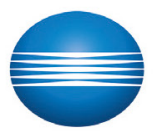

KONICA MINOLTA 

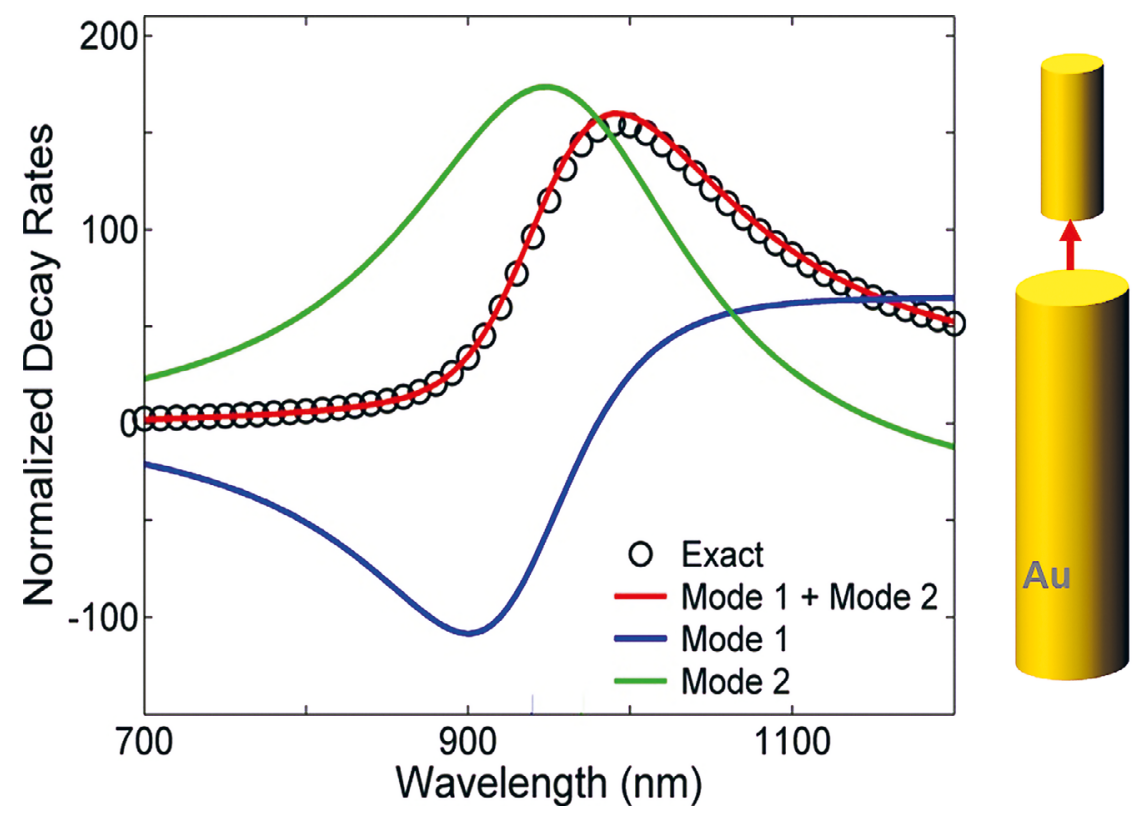

dans l'approximation hermitienne [2]. Ces prédictions nécessitent néanmoins de recevoir une confirmation expérimentale.

Intuitivement, on pourrait penser que la physique des microcavités à faibles pertes est essentiellement hermitienne, contrairement à celle des nanocavités plasmoniques. Ce partitionnement intuitif n'est pas toujours net. Par exemple, la théorie hermitienne de perturbation des cavités prédit très bien le décalage de fréquence introduit par la perturbation mais est complètement inutilisable pour prédire la variation de $Q$. Autre exemple, des résultats récents obtenus pour des cavités couplées de grands $Q$ montrent des profils spectraux de LDOS très fortement non-lorentziens, avec un caractère Fano aussi marqué que celui de la figure 3.

\section{CONCLUSION}

Le confinement extrême de la lumière dans de minuscules cavités optiques offre de nouvelles opportunités en nanosciences, pour étudier les interactions d'un faible nombre de photons avec des molécules individuelles, ou en nanotechnologie pour repousser les limites de la miniaturisation. Il pose également des questions fondamentales sur la nature du confinement. Résultats théoriques, outils numériques et effets physiques se combinent et remettent en question de nombreux préceptes acquis dans le cadre de la physique hermitienne.

Grâce à la mise en circulation des premiers logiciels, les progrès sont rapides. Par exemple, il y a quelques années, des calculs intensifs étaient conduits sur des super-ordinateurs pour comprendre l'effet de la rugosité sur le facteur de qualité des cavités à cristaux photoniques ou sur le transport de la lumière lente dans les guides périodiques. Aujourd'hui, ces mêmes prédictions pourraient être élégamment et rapidement obtenues en utilisant la théorie non-hermitienne de perturbation des cavités. Les progrès permettent aussi de multiplier et diversifier les travaux. Ainsi, les travaux applicatifs récents portent sur des sujets aussi variés que la génération de signaux non-linéaires avec des nano-résonateurs, ou l'étude d'objets individuels et de leur chimie réactionnelle grâce à des cavités hybrides mixant résonance électromagnétique et plasmonique, ou encore l'établissement d'une méthode systématique pour paramétriser les effets des corrections quantiques sur les conditions de passage des champs aux interfaces des nano-résonateurs. $\bullet$

\section{RÉFÉRENCES}

[1] F. Benz et al., Single-molecule optomechanics in "picocavities", Science 354, 726 (2016)

[2] P. Lalanne, W. Yan, K. Vynck, C. Sauvan, J.-P. Hugonin, Light interaction with photonic and plasmonic resonances, Laser Photonics Rev. 12, 1700113 (2018)

[3] R. Petit, Electromagnetic theory of gratings, Spinger-Verlag Berlin (1980)

[4] J.D. Jackson, Classical Electrodynamics, J. Wiley and Sons eds., New York (1974)

[5] E.M. Purcell, Spontaneous emission probabilities at radio frequencies, Phys. Rev. 69, 681 (1946)

[6] C. Sauvan, J.P. Hugonin, I.S. Maksymov, P. Lalanne, Theory of the spontaneous optical emission of nanosize photonic and plasmon resonators, Phys. Rev. Lett 110, 237401 (2013) 